\title{
DER ABWEICHENDE VERLAUF DES DUCTUS ARTE- RIOSUS BOTALLI EINES NEUGEBORENEN HIRSCHES
}

\author{
VON \\ Dr. A. J. VAN DAM \\ Zoologisches Laboratorium, Amsterdam
}

Im zoologischen Garten zu Amsterdam wurde am sechszehnten April 1939 von einer Hirschkuh der Art Rusa hippelaphus Cuv. ein Junges geworfen, das schon am anderen Tage wieder starb.

$\mathrm{Da}$ dieses Tier nur einen Tag gelebt hatte, war ein bedeutender Ductus arteriosus Botalli mit ansehnlichem Lumen zu erwarten, wie er auch in Kälbern ziemlich lange nach der Geburt erhalten bleibt.

Bei der Sektion zeigte sich tatsächlich ein ziemlich starker Ductus arteriosus mit deutlicher Höhlung. Dennoch war dieser Ductus arteriosus lange nicht so stark als er normal in derart jungen Tieren gefunden wird, ausserdem lag die distale Mündung dieses Gefässes nicht an der Aorta descendens sondern an der linken'Arteria subclavia (Abb. I).

Aus dem Aortabogen entsprangen bei diesem Tiere zwei Gefässe, als erstes der Truncus brachiocephalicus, der sich mehr cranialwärts teilte in die Arteria subclavia dextra und den Ductus bicaroticus, als zweites die selbständige Arteria subclavia sinistra.

Aus der Embryologie ist bekannt, dass bei Säugetieren die Arteria subclavia sinistra sich während der Entwicklung an den Aortenwurzel verschiebt. Ihre Abgangsstelle liegt erst caudalwärts vom Ductus Botalli, in späteren Stadien gegenüber dessen Einmündungsstelle und noch später cranialwärts von derselben. Bei Hirschen und Rindern setzt sich diese Verschiebung noch weiter kopfwärts fort, die Arteria subclavia sinistra schliesst sich an den Truncus brachiocephalicus an, so dass schliesslich von dem Arcus aortae nur ein Stamm cranialwärts zieht, die Aorta anterior.

Hammond untersuchte die embryonale Entwicklung des Kalbes und fand, dass beim Durchschnittembryo von $17 \mathrm{~mm}$ Länge der Arcus aortae und seine Seitenzweige alle schon in derselben Lage wie beim erwachsenen Tiere angeordnet sind ausser der Arteria subclavia sinistra. Diese hat ihren Ursprung aus der Aorta dann noch bedeutend hinter dem Ductus arteriosus Botalli. Während das Embryo von $17 \mathrm{~mm}$ heranwächst bis zu $22 \mathrm{~mm}$, verschiebt sich 
die Arteria subclavia sinistra an den Aortenwurzel bis zu ihrer definitiven Lage an der Aorta anterior.

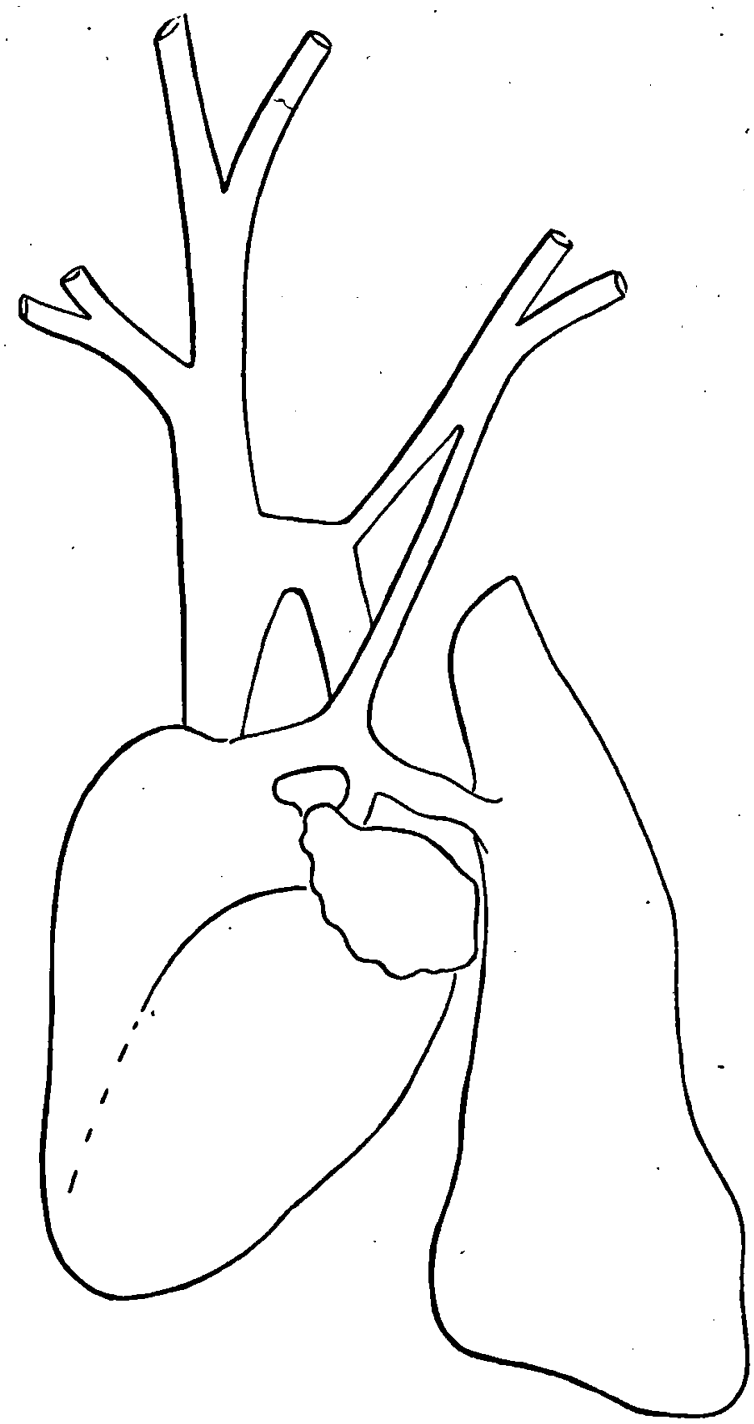

Abb. I. Rusa hippelaphus Cuvier. Herz und linke Lunge, Arcus aortae mit Seitenzweigen, Arteria pulmonalis und Ductus arteriosus Botalli (bei diesem Tiere verlaufend von A. pulmonalis zu Arteria subclavia sinistra).

Wir können ruhig annehmen, dass beim Hirschembryo die Entwicklung in ähnlicher Weise vor sich geht.

Die Abweichungen bei der jungen Rusa hippelaphus nun sind nicht einfach 
zu erklären durch die Annahme, dass die Arteria subclavia sinistra ihren Anschluss an die Aorta anteriọr nicht erreicht habe.

Man könnte sich aber vorstellen (Abb. 2 a-f): die Arteria subclavia sinistra habe ihre. Reise cranialwärts der Aorta entlang normal angefangen. Nachdem sie in gleicher Höhe angelangt war als die Einmündungsstelle des Ductus Botalli, habe sie sich an diesen Ductus Botalli angeschlossen und sich zusammen mit ihm von der Aorta losgerissen.

In ihrer späteren, mehr cranialwärts ger:chteten Lage habe sie den Ductus Botalli mit sich gezogen, während sie durch ihre Verbindung mit diesem Ductus und durch ihn mit der Arteria pulmonalis daran verhindert war, ihren Anschluss an die Aorta anterior zu vollführen (Abb. I).

Gelegenheit mehrere Herze von Rusa hippelaphus zu untersuchen bot sich mir keine. Wohl aber war ich im Stande einige Herze von Odocoileus-Arten zu besehen, was ich der Höflichkeit des Herrn Wagenaar Hummelinck verdanke, und einige Exemplare von jungen Cervidae aus der Sammlung des zoologischen Museums zu Amsterdam zu betrachten, was Herr Direktor DE BEAUFORT mir möglich machte. Beiden möchte ich hier meinen besten Dank sagen.

Bei allen diesen Tieren war der Verlauf des Ductus arteriosus Botalli vollkommen normal. Auch war die Arteria subclavia sinistra immer ein Seitenzweig der Aorta anterior. Nur die Verzweigung dieser Aorta anterior zeigte mehrere Variationen.

Nach Parsons gehen beim Geschlecht Cervus. in $1 / 4$ der Fälle die beiden Arteriae subclaviae ungefähr in gleicher Höhe vom gemeinsamen Truncus ab und ein ziemlich langer Truncus bicaroticus bleibt übrig, der zich zum Schlusse in die beiden Carotiden teilt. In $3 / 4$ der Fälle zweigt sich zuerst die linke Arteria subclavia ab, dann die rechte Arteria subclavia, der Truncus bicaroticus teilt sich nach einem ziemlich langen Verlauf in die beiden Carotiden.

Lassila findet bei einem Exemplar von Cervus dama eine Aorta anterior, die als erste die Arteria subclavia sinistra entsendet und ein wenig weiter fast gleichzeitig 4 Gefässe aussendet, als erste die Arteria vertebralis dextra, dann die A. subclavia dextra und dann die beiden Arteriae carotides. Alle diese Anfangsstellen liegen so nahe beisammen, dass ein deutlicher Truncus bicaroticus nicht nach zu weisen ist. (LAssila, Fig. 15. Die Arteria vertebralis dextra und die A. subclavia dextra haben hier zufälligerweise keinen gemeinschaftlichen Ursprung.)

Bei den von mir betrachteten Herzen kommen beide Fälle von PArsons (Abb. 3 und Abb. 4) wie auch der Fall Lassila's vor (Abb. 5 und Abb. 7). Bei den Odocoileus-Exemplaren Od. 3 I und Od. 23 aber, wo ebenfalls die Arteria subclavia dextra und die beiden Carotiden ungefähr an gleicher Stelle aus dem gemeinsamen Truncus abzweigen, ist es die Arteria carotis sinistra, die sich als erste abzweigt, wenn auch der Abstand klein ist (Abb. 6). 


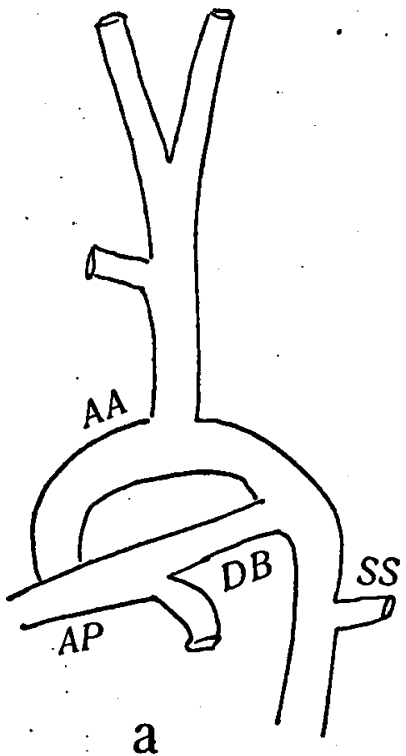

a
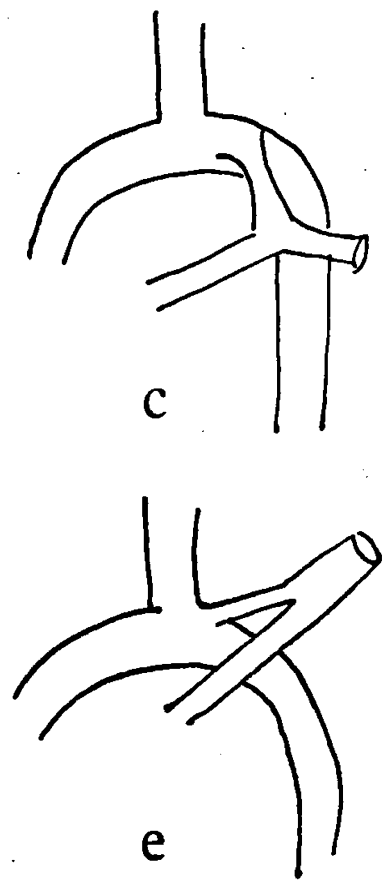
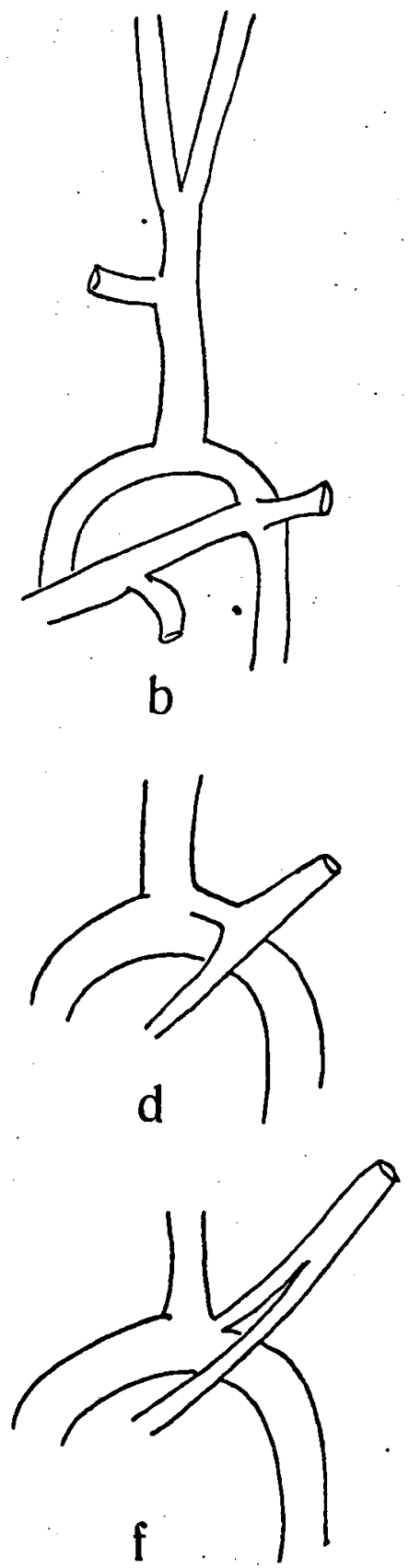

Abb. 2. Schema der möglichen embryonalen Veränderungen - vom normalen jungen Stadium a bis zum Stadium $f$, das übereinstimmt mit $\mathrm{Abb}$. I. AA Arcus aortae. AP Arteria pulmonalis. DB Ductus Botalli. SS Subclavia sinistra. 
In Abb. 8 und mehr noch in Abb. 9 sieht man wie nach Abgabe der Arteria subclavia sinistra und der Arteria carotis sinistra ein deutlicher gemeinsamer Stamm für die A. subclavia dextra und die A. carotis dextra, also eine Arteria anonyma übrig bleibt.

In dem Falle von Abb. Io ist der Abstand zwischen den Ursprüngen der A. subclavia sinistra und der A. carotis sinistra fast gleich lang wie die Arteria anonyma.

So zeigt sich, dass in den wenigen von mir untersuchten Herzen alle Übergänge sich finden vom Falle der $\mathrm{Abb}$. 3 bis $z u$ dem der Abb. Io. Die drei Exemplare, die eine deutliche Arteria anonyma zeigen, sind alle aus Suriname geburtig. Leider sind sie nicht determiniert worden. Die Determination solcher jungen Tiere ist wohl sehr beschwerlich.

Hier folgt die kurze Beschreibung der von mir besehenen Herze, in welcher nur der Ductus arteriosus Botalli und die Verzweigung der Aorta anterior genannt werden.

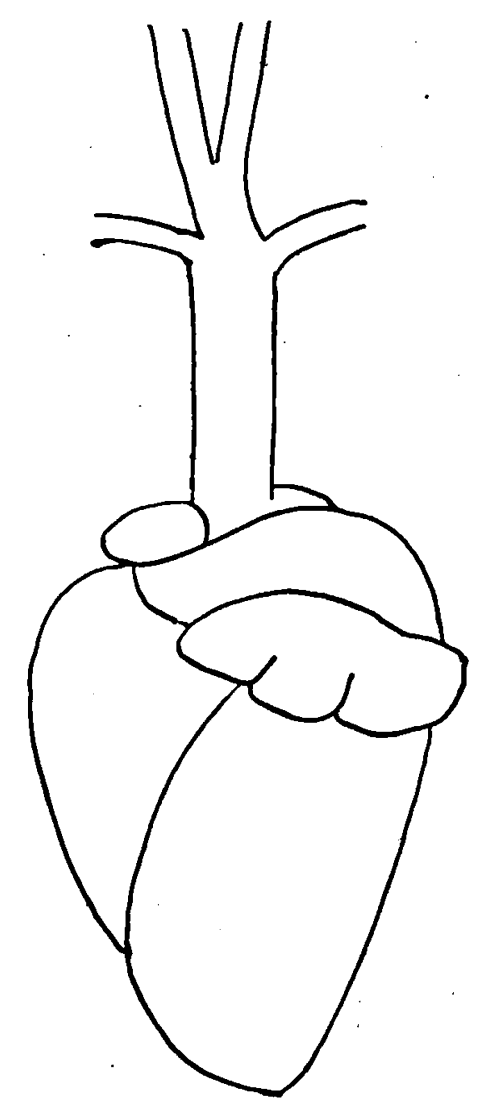

Abb. 3. Alces machlis Ogilby. Herz und Verzweigung der Aorta anterior.
Alces machlis Ogilby.

Foetus von sechs Monaten. Abb. 3 .

Der Ductus arteriosus Botalli ist sehr stark und hat einen normalen Verlauf. Von der Aorta anterior zweigen sich fast gleichzeitig die linke und rechte Arteria subclavia ab. Ein kurzer Truncus bicaroticus teilt sich in die beiden Carotiden.

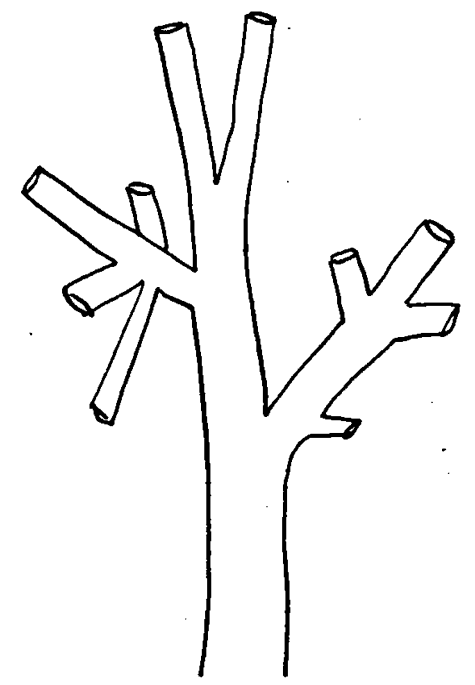

Abb. 4. Muntiacus (Cervulus) muntjak Zimm. Verzweigung der Aorta anterior. 
Muntiacus (Cervulus) muntjak Zimm.

Erwachsenes $0^{\circ}$. Abb. 4.

Das Ligamentum arteriosum ist klein und normal verlaufend. Die Aorta anterior gibt ab die Arteria subclavia sinistra und verfolgt ihren Weg als deutlicher I $\mathrm{cm}$ langer Truncus brachiocephalicus. Von ihm zweigt sich ab die Arteria subclavia dextra. Der Truncus bicaroticus ist etwa gleich lang als der Truncus brachiocephalicus.

Hirschfoetus Rioeng, Nordküste Flores, 12 ${ }^{\circ}$ O.L. Abb. 5. Das Alter dieșes Tieres ist unbekannt. Die Länge vom Kopfe (frontal) bis zur Schwanzwurzel ist mehr als $30 \mathrm{~cm}$.

Der Ductus arteriosus Botalli sehr stark mit normalem Verlauf. Als erste zweigt sich von der Aorta anterior ab die linke Arteria subclavia, dann die rechte A. subclavia und gleich darauf folgt die Gabelung der Carotiden.

Odocoileus gymnotus gymnotus (Wiegm.) ס".

Die Eltern waren importiert aus Sucre, Venezuela (Od. 3I Hummelinck 1940, 2, p. 89). Alter 2 Tage. Abb. 6.

Der Ductus arteriosus Botalli ist sehr stark und hat einen normalen Verlauf. Von der Aorta anterior zweigt sich als erste die Arteria subclavia sinistra ab. Nach einem ziemlich langen Verlauf teilt sich der Truncus brachiocephalicus fast gleichzeitig in drei Zweige. Die Arteria carotis sinistra ist aber deutlich

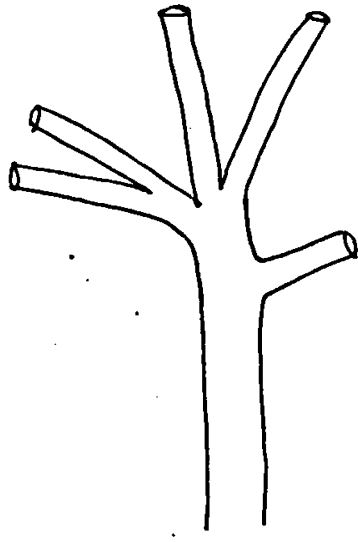

Abb. 5. Hirsch-Foetus Nordküste Flores. Verzweigung der Aorta anterior. der erste dieser $Z$ weige, sodass wir von einer sehr kurzen Arteria anonyma reden können.

Odocoileus gymnotus gymnotus (Wiegm.).

Erwachsenes 07 . Die Eltern waren aus Sucre, Venezuela, importiert. (Od. 27 Hummelinck 1940, 2, p. 89).

Ligamentum arteriosum klein, normal verlaufend. Die Aorta anterior entsendet als ersten Seitenzweig die Arteria subclavia sinistra. Gleich nach dieser Abzweigung ist der Truncus brachiocephalicus abgeschnitten.

Odocoileus gymnotus gymnotus (Wiegm.).

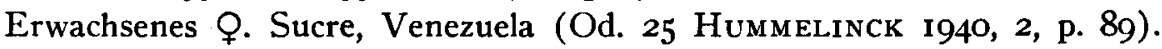
Ligamentum arteriosum sehr kurz und in normaler Lage. Als erster Seitenzweig der Aorta anterior zeigt sich die Arteria subclavia sinistra, nach einem ziemlich kurzen Verlauf zweigt sich eine nicht sehr starke Arteria ab, wahrscheinlich die Arteria carotis sinistra. Gleich neben dem Ursprung dieser Arteria ist der Truncus brachiocephalicus schief nach unten abgeschnitten, Bijdragen tot de Dierkunde, Afl. 28. 
sodass die Abgangsstellen der Arteria subclavia dextra und der Arteria carotis dextra nicht $z \mathbf{z}$ sehen und selbst nicht zu vermuten sind.

Eine Möglichkeit der Verzweigung wie bei Od. 31 Abb. 6 ist aber sehr gut denkbar.

Odocoileus gymnotus gymnotus (Wiegm.).

Erwachsenes $\sigma^{7}$. Sucre, Venezuela (Od. 23 Hummelinck I940, 2, p. 89).

Das Ligamentum arteriosum ist sehr klein und hat einen normalen Verlauf. Von der Aorta anterior zweigt sich als erste ab die Arteria subclavia sinistra.

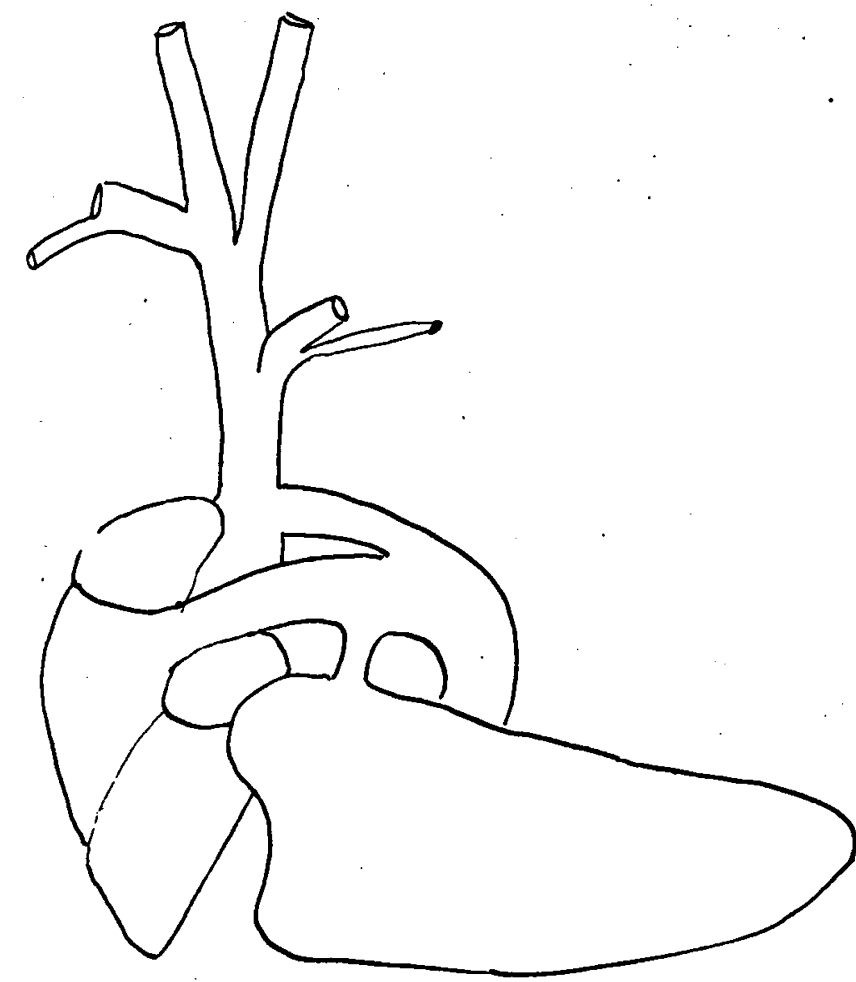

Abb. 6. Odocoileus gymnotus gymnotus (Wiegm.). Herz und linke Lunge mit den zwei grossen Arterien, so auseinander gelegt, dass der starke Ductus arteriosus Botalli zu sehen ist. Verzweigung der Aorta anterior.

Nach einem ziemlich langen Verlauf teilt sich der Truncus brachiocephalicus auf in eine Arteria carotis sinistra und eine kurze Arteria anonyma. Die Anfangsstellen der Arteria subclavia dextra und der Arteria carotis dextra sind noch gerade erhalten geblieben.

Odocoileus gymnotus gymnotus (Wiegm.) o Die Eltern waren importiert aus Sucre Venezuela. Alter 5 Monate. (Od. 30 Hummelinck 1940, 2, p. 89). 
Das Ligamentum arteriosum hat einen normalen Verlauf. Die Aorta anterior entsendet als erste die Arteria subclavia sinistra, nach einem ziemlich langen Verlauf die Arteria carotis sinistra. Gleich neben deren Ursprungsstelle ist die Arteria anonyma abgeschnitten.

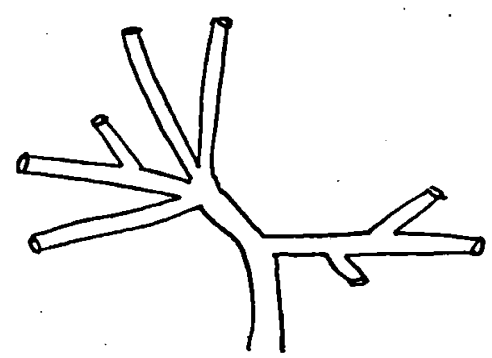

Abb. 7. Foetus eines surinamischen Hirsches. Verzweigung der Aorta anterior.

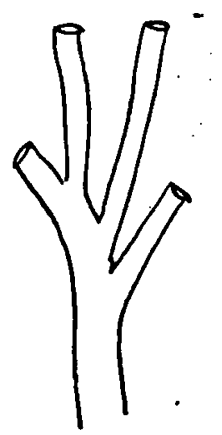

Abb. 8. Cervus spec. neonatus. Suriname.

E. A. Polak don. Verzweigung der Aorta anterior.

Odocoi'eus gymnotus curassavicus Hummelinck.

Curaçao. (Od. 2 Hummelinck I940, I, p. 65). Alter 9 Monate.

Ligamentum arteriosum Botalli klein mit normalem Verlauf. Von der Aorta

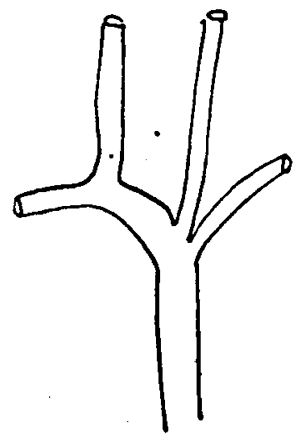

Abb. 9. Cervus spec. neonatus. Suriname. v. Brussel leg. Verzweigung der Aorta anterior.

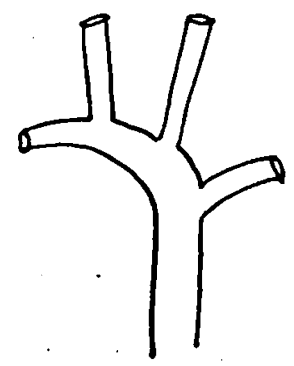

Abb. 10. Cervus spec. Foetus. Suriname. v. Brussel leg. Verzweigung der Aorta anterior.

anterior zweigt sich als erste ab die Arteria subclavia sinistra. Der Truncus brachiocephalicus ist ziemlich kurz abgeschnitten. Die wetere Verżweigungen sind nicht mehr zu sehen. 
Odocoileus gymnotus curassavicus Hummelinck.

Erwachsenes $\sigma^{7}$. Curaçao. (Od. 5 Hummelinck 1940, I, p. 65).

Das Ligamentum arteriosum hat einen normalen Verlauf. Die Aorta anterior entsendet die Arteria subclavia sinistra. Der Truncus brachiocephalicus ist kurz abgeschnitten. Weitere Verzweigungen sind also nicht mehr zu sehen.

Surinamischer Hirsch.

ơ. Foetus. Abb. 7.

Länge vom Kopf (frontal) bis zur Schwanzwurzel $17 \mathrm{~cm}$.

Der Ductus arteriosus Botalli ist sehr stark und hat einen normalen Verláuf. Von der Aorta anterior zweigt sich als erste die Arteria subclavia sinistra $a b$ und dann folgen etwa an gleicher Stelle die Abzweigungen der beiden Carotiden und der Arteria subclavia dextra, die sich sofort wieder in drei Zweige teilt.

Cervus spec.

§. Suriname. Abb. 8.

Alter unbekannt, wahrscheinlich neonatus.

Der Ductus arteriosus Botalli hat einen normalen Verlauf und ist sehr kräftig. Die Abzweigungsstellen der 4 Hauptarterien aus der Aorta anterior liegen einander nahe. Als erste biegt sich ab die Arteria subclavia sinistra, dann die Arteria carotis sinistra und schliesslich scheiden sich die Arteria subclavia dextra und die Arteria carotis dextra.

Cervus spec.

Q. Suriname. Neonatus. Abb. 9.

Ductus arteriosus Botalli sehr stark mit normalem Verlauf. Aus der Aorta anterior zweigen sich nebeneinander ab die Arteria subclavia sinistra, die Arteria carotis sinistra und eine Arteria anonyma, die sich erst $7 \mathrm{~mm}$ weiter in die Arteria subclavia dextra und die Arteria carotis dextra teilt.

Cervus spec.

ơ. Suriname. Abb. Io.

Wahrscheinlich noch Foetus.

Ductus arteriosus stark und mit normalem Verlauf. Erst zweigt sich die Arteria subclavia sinistra von der Aorta anterior ab, etwa $3 \mathrm{~mm}$ weiter die Arteria carotis sinistra und wiederum einige $\mathrm{mm}$ weiter liegt die Verzweigung in Arteria subclavia dextra und Arteria carotis dextra.

\section{LITERATUR}

Golub, D. M., 193I. Die Entwicklung des Aortenbogens und der von ihm ausgehenden Äste bei Säugetieren. I Entwicklung der Kiemenbogen bei Kalb und Menschen. Z. Anat. Entw. gesch. Bd. 95, p. 447.

Hafferl, A., 1933. Das Arteriensystem. Handb. d. vergl. Anat. d. Wirbeltiere, L. Bolk, E. Göppert, E. Kallius, W. Lubosch. Bd. 6, p. 563. 
Hammond, W. S., 1938. The developmental transformations of the aortic arches in the calf (Bos taurus) with especial reference to the formation of the arch of the aorta. Am. Jour. Anat. Vol. 62, p. I49.

Hochstetter, F., 1890. Uber die Entwicklung der A. vertebralis beim Kaninchen, nebst Bemerkungen über die Entstehung der Ansa Vieussenii. Morph. Jahrb. Bd. 16, p. 572.

Hummelinck, P. Wagenaar, 1940. (I) Studies on the fauna of Curaçao, Aruba, Bonaire and the Venezuelan islands. Dissertation Utrecht.

-, 1940. (2) Mammals of the genera Odocoileus and Sylvilagus. Stud. Faun. Cur. Ar. Bon. Venez. isl. Vol. 2, No. 6, p. 83.

Lassila, V., 1928. Der Aortenbogen und die sich von ihm abzweigenden grossen Arterienstämme nebst deren Verhältnis zu den Nervi sympaticus, vagus und recurrens bei den Säugern. Ann. Acad. Scient. Fennicae Tom. 27, p. I.

Parsons, F. G., I902. On the arrangement of the branches of the mammalian aortic arch. Jour. Anat. Phys. Vol. 36 (n.s. Vol. 16). 